\title{
Dark-soliton-like magnetic domain walls in a two-dimensional ferromagnetic superfluid
}

\author{
Xiaoquan $\mathrm{Yu} \odot^{1,2, *}$ and P. B. Blakie ${ }^{2, \dagger}$ \\ ${ }^{1}$ Graduate School of China Academy of Engineering Physics, Beijing 100193, China \\ ${ }^{2}$ Department of Physics, Centre for Quantum Science, and Dodd-Walls Centre for Photonic and Quantum Technologies, \\ University of Otago, Dunedin, New Zealand
}

(Received 18 August 2020; accepted 23 March 2021; published 14 April 2021)

\begin{abstract}
We report a stable magnetic domain wall in a uniform ferromagnetic spin-1 condensate, characterized by the magnetization having a dark soliton profile with nonvanishing superfluid density. We find exact stationary solutions for a particular ratio of interaction parameters with and without magnetic fields, and develop an accurate analytic solution applicable to the whole ferromagnetic phase. In the absence of magnetic fields, this domain wall relates various distinct solitary excitations in binary condensates through $\mathrm{SO}(3)$ spin rotations, which otherwise are unconnected. Remarkably, studying the dynamics of a quasi-two-dimensional (quasi-2D) system we show that standing wave excitations of the domain wall oscillate without decay, being stable against the snake instability. The domain wall is dynamically unstable to modes that cause the magnetization to grow perpendicularly while leaving the domain wall unchanged. Real-time dynamics in the presence of white noise reveals that this "spin twist" instability does not destroy the topological structure of the magnetic domain wall.
\end{abstract}

DOI: 10.1103/PhysRevResearch.3.023043

\section{INTRODUCTION}

A domain wall is a nonlinear excitation that interpolates between two different ground states, playing an important role in both equilibrium and out-of-equilibrium phase transitions with discrete symmetry breaking [1-3]. It appears in broad fields of physics, ranging from statistical mechanics [1] and quantum field theories [2,3] to cosmology [4].

Bose-Einstein condensates (BECs) provide a platform to study various topological excitations including vortices, domain walls, and solitons. Unlike vortices, a wide class of domain walls and solitons are unstable to the so-called snake instability in two-dimensional (2D) systems, when the size of the system is larger than the width of domain walls/solitons. Examples include dark solitons [5-8], phase domain walls [9-11], magnetic solitons [12-14], and nematic domain wall-vortex composites [15], in scalar, coherently coupled, binary, and antiferromagnetic spin-1 BECs, respectively. An outstanding challenge is thus to obtain stable 2D domain walls/solitons, which would open the door to studying their rich dynamical properties.

Thanks to the $\mathrm{U}(1)$ gauge symmetry and the rotational $\mathrm{SO}(3)$ symmetry, a spin-1 ferromagnetic BEC exhibits both superfluid and magnetic order quantified by the superfluid density and the magnetization [16-20], respectively. It offers

\footnotetext{
*xqyu@gscaep.ac.cn

†blair.blakie@otago.ac.nz
}

Published by the American Physical Society under the terms of the Creative Commons Attribution 4.0 International license. Further distribution of this work must maintain attribution to the author(s) and the published article's title, journal citation, and DOI. an opportunity to explore magnetic domain walls (interfaces separating oppositely magnetized regions) absent in scalar, binary, and antiferromagnetic BECs. Most work in ferromagnetic spin-1 BECs has focused on spin textures and their nonequilibrium dynamics (e.g., [21-29]). The domain wall physics remains largely unexplored and very little is known about their structures, stability in high dimensions, and potential connections to vector solitons [30-32].

In this paper, we present an analytic solution of a stable magnetic domain wall in a quasi-2D spin-1 ferromagnetic BEC, characterized by magnetization $\mathbf{F}$ having the typical profile of a dark soliton [Fig. 1(a)]: a $\pi$-phase (direction of F) jump crossing the domain wall and $\mathbf{F}=\mathbf{0}$ at the center, breaking the $\mathbb{Z}_{2}$ symmetry. In contrast to most domain walls/solitons in BECs, the magnetic domain wall is stable against the snake instability in two dimensions. This is verified by studying transverse standing waves on these domain walls, finding they oscillate without decay. Instead, the system has a linear dynamic instability driven by modes localized near the domain-wall core that cause a growth of the perpendicular components of the magnetization. The resulting spin texture corresponds to a chain of spin vortex-antivortex pairs along the domain wall. Real-time dynamics in the presence of white noise shows that the magnetic domain wall survives. Exact solutions are obtained for a large spin-dependent interaction strength $g_{s}$ with and without magnetic fields. These exact solutions are distinct from two well-known solvable cases: the Manakov regime [33] ( $g_{s}=0$ in spin-1 BECs) and the magnetic soliton regime (constant number density) [12]. In the absence of magnetic fields, $\mathrm{SO}(3)$ spin rotations relate a family of degenerate solutions, and we show that for particular rotations the underlying component wave function can map onto a range of solitons and domain walls proposed for binary condensates. Thus, a distinct set of unrelated nonlinear 

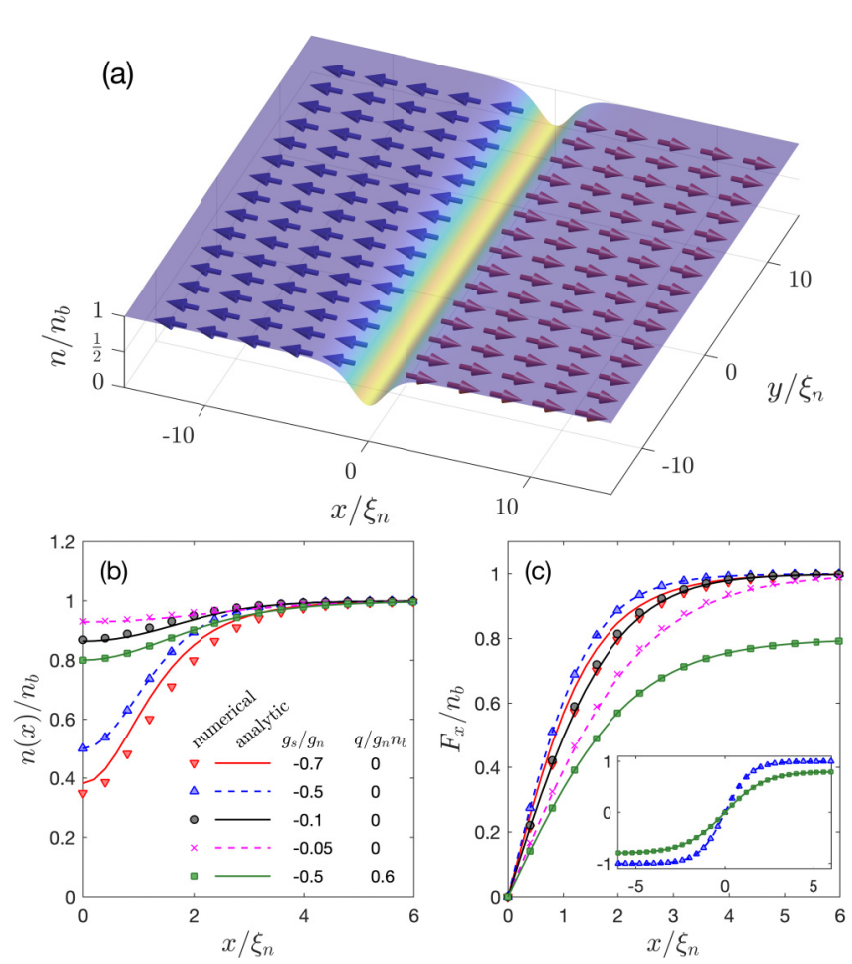

FIG. 1. (a) Schematic of a transverse magnetic domain wall (along $y$ axis) in a system of background density $n_{b}$. The arrows represent the transverse magnetization vector $\left(F_{x}, F_{y}\right)$, and the background color shows the superfluid density. A comparison between analytical predictions (lines) and numerical results (symbols) for the (b) density and (c) $F_{x}$ spin density for various values of $g_{s} / g_{n}$ and $q$. The inset shows two complete profiles of $F_{x}$ at the exactly solvable point with $q=0$ and $q \neq 0$, respectively. Here, $\xi_{n}=\hbar / \sqrt{M g_{n} n_{b}}$ is the density healing length.

excitations are found to be contained within our solution, unified by its symmetries.

\section{FORMALISM FOR A SPIN-1 BEC}

The Hamiltonian density of a quasi-2D spin-1 BEC [34] reads

$\mathcal{H}=\frac{\hbar^{2}|\nabla \psi|^{2}}{2 M}+\frac{g_{n}}{2}\left|\psi^{\dagger} \psi\right|^{2}+\frac{g_{s}}{2}\left|\psi^{\dagger} \mathbf{S} \psi\right|^{2}+q \psi^{\dagger} S_{z}^{2} \psi$,

where the three-component wave function $\psi=$ $\left(\psi_{+1}, \psi_{0}, \psi_{-1}\right)^{T}$ describes the condensate amplitude in the $m=+1,0,-1$ sublevels, respectively. Here, $M$ is the atomic mass, $g_{n}>0$ is the density interaction strength, $g_{s}$ is the spin-dependent interaction strength, $\mathbf{S}=\left(S_{x}, S_{y}, S_{z}\right)$ with $S_{v=x, y, z}$ being the spin-1 matrices [35], and $q$ denotes the quadratic Zeeman energy. The spin-dependent interaction term allows for spin-mixing collisions in which two $m=0$ atoms collide and convert into $m=+1$ and -1 atoms, and the reverse process.

The dynamics of the field $\psi$ is given by the GrossPitaevskii equations (GPEs) $i \hbar \partial \psi / \partial t=\delta \mathcal{H} / \delta \psi^{\dagger} \equiv \mathcal{L}_{\mathrm{GP}} \psi$, which in component form is

$$
\begin{aligned}
i \hbar \frac{\partial \psi_{ \pm 1}}{\partial t} & =\left[H_{0}+g_{s}\left(n-2 n_{\mp 1}\right)+q\right] \psi_{ \pm 1}+g_{s} \psi_{0}^{2} \psi_{\mp 1}^{*}, \\
i \hbar \frac{\partial \psi_{0}}{\partial t} & =\left[H_{0}+g_{s}\left(n_{+1}+n_{-1}\right)\right] \psi_{0}+2 g_{s} \psi_{0}^{*} \psi_{+1} \psi_{-1},
\end{aligned}
$$

where $H_{0}=-\hbar^{2} \nabla^{2} / 2 M+g_{n} n$, with $n=\sum_{m} n_{m}$ and $n_{m}=$ $\left|\psi_{m}\right|^{2}$ being the total and component densities, respectively. Spin-1 BECs exhibit magnetic order, e.g., the magnetization $\mathbf{F} \equiv \psi^{\dagger} \mathbf{S} \psi$ [36] is the order parameter of ferromagnetic phases $|\mathbf{F}|>0$ for $g_{s}<0\left({ }^{87} \mathrm{Rb}\right.$ or $\left.{ }^{7} \mathrm{Li}\right)$. In contrast, antiferromagnetic phases with $g_{s}>0\left({ }^{23} \mathrm{Na}\right)$ have $\mathbf{F}=0$. In the absence of magnetic fields, i.e., $q=0, \mathcal{H}$ is invariant under $\mathrm{SO}(3)$ spin-rotations and the total magnetization $\int d^{2} \mathbf{r} \mathbf{F}$ is conserved.

\section{DARK-SOLITON-LIKE MAGNETIC DOMAIN WALLS}

For a uniform ferromagnetic system with total density $n_{b}$ and at $q=0$, the energy density $\mathcal{H}=g_{n} n_{b}^{2} / 2+g_{s}|\mathbf{F}|^{2} / 2$ is minimized for states with $|\mathbf{F}|=n_{b}$. The chemical potential is $\mu=\left(g_{n}+g_{s}\right) n_{b}$. We search for a straight-line domain wall connecting the two distinct magnetic ground states characterized by $\mathbf{F}= \pm n_{b} \hat{\mathbf{e}}$, where $\hat{\mathbf{e}}$ is a $3 \mathrm{D}$ unit vector along an arbitrary direction [see Fig. 1(a)]. For convenience, the domain wall is chosen parallel to the $y$ axis and the core is located at $x=0$. We find a solution of the general form

$$
\mathbf{F} \simeq n(x) \tanh (x / \ell) \hat{\mathbf{e}},
$$

where $\ell=\hbar / \sqrt{4\left|g_{s}\right| M n_{b}}$. This result is exact for a particular set of interaction parameters, and a good approximation in general, as we discuss further below. This domain wall is of the Ising type, rather than the Bloch or Néel type, signified by $\mathbf{F}$ vanishing at the core and changing its sign across the core. The solution (3) has the characteristic profile of dark soliton and we refer to it as dark-soliton-like magnetic domain wall (MDW). This domain wall is in magnetic order but not in the superfluid order, i.e., the superfluid density $n(x)$ does not vanish, but has a dip at the core to minimize the energy.

\section{A. Exact solutions}

When the width of the density dip coincides with $\ell$, occurring at $g_{s}=-g_{n} / 2$ [37], Eq. (2) admits an exact solution

$$
\mathbf{F}(x)=n_{b} \tanh \left(\frac{x}{2 \ell}\right) \hat{\mathbf{e}}, \quad n(x)=n_{b}\left[1-\frac{1}{2} \operatorname{sech}^{2}\left(\frac{x}{2 \ell}\right)\right] .
$$

This system has a $\mathrm{SO}(3)$ symmetry, which relates a continuous family of degenerate MDW solutions connected by $\mathrm{U}(1)$ gauge and spin $\mathcal{U}(\alpha, \beta, \tau)=e^{-i \alpha S_{z}} e^{-i \beta S_{y}} e^{-i \tau S_{z}}$ rotations, where $\{\alpha, \beta, \tau\}$ are the Euler angles. We illustrate three members of this family in Table I: (i) For the case of an $F_{x}$ domain wall [i.e. $\hat{\mathbf{e}}=\hat{\mathbf{x}}$ ], the underlying wave functions can have two distinct vector-soliton profiles, and the corresponding stationary GPE can be mapped onto that of a miscible binary BEC. (ii) A sine-Gordon-type soliton (SGS) of the phase difference $\theta_{d} \equiv \theta_{ \pm 1}-\theta_{0}$, where $\psi_{m}=\left|\psi_{m}\right| e^{i \theta_{m}}$. A SGS has been predicted to exist in a coherently-coupled binary BEC, with dynamics mimicking processes in quantum 
TABLE I. Component representation of the MDW after various spin rotations. Vector soliton sector: type-I vector soliton is chosen as a reference point. In this presentation, the reduced GPEs are related to a miscible binary system and becomes decoupled at $g_{s}=-g_{n} / 2$, allowing the exact solution. SGS: $\theta_{d}$ satisfies the sine-Gordon equation. Binary domain-wall sector: The reduced GPEs describe an immiscible binary system and the corresponding exact solution coincides with a solution discussed in a different context [40].

\begin{tabular}{|c|c|c|c|}
\hline $\begin{array}{l}(\alpha, \beta, \tau) \\
\mathrm{U}(1)\end{array}$ & $\begin{array}{l}\text { type-I: } 0 ; \text { type-II: }(\pi / 2,-\pi / 2,-\pi / 2) \\
\text { type-I: } 1 ; \text { type-II: } e^{i \pi / 2}\end{array}$ & $\begin{array}{c}(-\pi / 2,-\pi / 4,-\pi / 2) \\
e^{-i \pi / 4}\end{array}$ & $\begin{array}{c}(\pi / 2, \pi / 2,0) \\
e^{i 3 \pi / 2}\end{array}$ \\
\hline$\left.\psi\right|_{g_{s}=-g_{n} / 2}$ & $\begin{array}{l}\text { I: } \psi_{ \pm 1}=\sqrt{n_{b}} / 2 \tanh (x / 2 \ell) ; \psi_{0}=\sqrt{n_{b} / 2} \\
\text { II: } \psi_{ \pm 1}=\sqrt{n_{b}} / 2, \psi_{0}=\sqrt{n_{b} / 2} \tanh (x / 2 \ell)\end{array}$ & $\begin{aligned} \psi_{ \pm 1} & =\sqrt{n_{ \pm 1}} e^{i \theta_{d} / 2}, \psi_{0}=\sqrt{n_{0}} e^{-i \theta_{d} / 2} \\
\theta_{d}(x) & =2 \arctan e^{x / \ell}, 2 n_{ \pm 1}=n_{0}=n / 2\end{aligned}$ & $\begin{array}{c}\psi_{ \pm 1}=\sqrt{n_{b}} / 2[1 \mp \tanh (x / 2 \ell)] \\
\psi_{0}=0\end{array}$ \\
\hline $\mathbf{F}$ & $F_{x}=n_{b} \tanh (x / 2 \ell)$ & $F_{x}=n_{b} \tanh (x / 2 \ell)$ & $F_{z}=-n_{b} \tanh (x / 2 \ell)$ \\
\hline GPE & $\begin{array}{c}0=\left[H^{\prime}+2 g_{n} n_{ \pm 1}+\left(g_{n}+2 g_{s}\right) n_{0}\right] \psi_{ \pm 1} \\
0=\left[H^{\prime}+g_{n} n_{0}+2\left(g_{n}+2 g_{s}\right) n_{ \pm 1}\right] \psi_{0} \\
H^{\prime}=-\frac{\hbar^{2}}{2 M} \partial_{x}^{2}-\mu\end{array}$ & $\begin{aligned} 0= & \frac{\hbar^{2}}{2 M} \partial_{x}\left(n \partial_{x} \theta_{d}\right)+g_{s} n^{2} \sin \left(2 \theta_{d}\right) \\
0= & \frac{\hbar^{2}}{2 M}\left[\frac{1}{2}\left(\partial_{x} \theta_{d}\right)^{2}-\frac{2}{\sqrt{n}} \partial_{x}^{2} \sqrt{n}\right] \\
& +2\left[n\left(g_{n}+g_{s} \cos ^{2} \theta_{d}\right)-\mu\right]\end{aligned}$ & $\begin{array}{l}0=\left[H^{\prime}+\left(g_{n}+g_{s}\right) n_{+1}+\left(g_{n}-g_{s}\right) n_{-1}\right] \psi_{+1} \\
0=\left[H^{\prime}+\left(g_{n}+g_{s}\right) n_{-1}+\left(g_{n}-g_{s}\right) n_{+1}\right] \psi_{-1}\end{array}$ \\
\hline Related & Vector soliton of a three-component BEC and a & sine-Gordon-type soliton, also realized & Density domain wall of an immiscible binary \\
\hline
\end{tabular}
miscible binary BEC

chromodynamics [9]. Here, the SGS can be produced by a spin rotation of the vector soliton in Table I and the nonlinear spin-mixing interaction provides the necessary couplings between the component phases, having the advantage that no external fields are required [38]. (iii) For an $F_{z}$ domain wall, the corresponding wave function coincides with a (density) domain wall of an immiscible binary BEC [39].

In the context of binary BECs, the vector solitons, the SGS, and the density domain wall are unrelated. In a spin-1 BEC, these distinct nonlinear excitations are unified by spin rotations of our MDW solution. With inadequate degrees of freedom and symmetries, such connection can not be made within the binary BEC $[19,41]$. However, it is important to note that the dynamics and stability properties of the MDW reveal the spin-1 nature and exhibit distinct behaviors from related excitations in binary systems (see below). A recent study on magnetic solitons in antiferromagnetic BECs has also explored the role of the rotational symmetry [42].

\section{B. Away from the exactly solvable point}

Away from the exactly solvable point, we develop a selfconsistent asymptotic analysis of the stationary GPEs at $x \gg$ $\ell$, combined with an account of the local core structure, and we find an accurate approximate form for the density,

$$
\frac{n(x)}{n_{b}} \simeq \begin{cases}\frac{\cosh (x / \lambda \ell)}{a_{1} \cosh (x / \lambda \ell)+a_{1}^{2} b_{1}}+1-\frac{1}{a_{1}}, & g_{s}<-\frac{g_{n}}{5} \\ 1+\frac{4 b_{1} g_{s}}{2\left(g_{n}+g_{s}\right) \cosh ^{2}(x / \ell)+g_{1}}, & -\frac{g_{n}}{5}<g_{s}\end{cases}
$$

where $\quad \lambda=\sqrt{-g_{s} /\left(g_{n}+g_{s}\right)}, \quad a_{1}=-\left(2 g_{n}^{2}+2 g_{n} g_{s}-g_{s}^{2}\right) /$ $\left(3 g_{s}\left(g_{n}+g_{s}\right)\right), \quad b_{1}=3\left(g_{n}+g_{s}\right) /\left(2 g_{n}+g_{s}\right), \quad$ and $\quad g_{1}=$ $2 b_{1}\left(2 g_{n}-5 g_{s}\right) / 3$.

In the following we show the procedure to obtain Eqs. (5). Specializing to the SGS (see Table I), we work with the hydrodynamical variables $\left\{n, n_{d}, \theta_{d}, \theta_{s}\right\}$, where $n_{d}=2 n_{ \pm 1}-n_{0}$, $\theta_{s}=\theta_{ \pm 1}+\theta_{0}, \theta_{+1}=\theta_{-1}$, and $n_{+1}=n_{-1}$. For a stationary state, the total number current $\mathbf{J}_{n}=\hbar^{2} /(2 M)\left(n \nabla \theta_{s}+n_{d} \nabla \theta_{d}\right)$ should vanish. Apparently $\theta_{s}=0$ and $n_{d}=0$ solve $\mathbf{J}_{n}=0$, and for this case Eqs. (2) [or Eqs. (A1)] reduce to

$$
\begin{aligned}
& 0=\frac{\hbar^{2}}{2 M} \partial_{x}\left(n \partial_{x} \theta_{d}\right)+g_{s} n^{2} \sin \left(2 \theta_{d}\right), \\
& \mu=\frac{\hbar^{2}}{4 M}\left[\frac{\left(\partial_{x} \theta_{d}\right)^{2}}{2}-\frac{2 \partial_{x}^{2} \sqrt{n}}{\sqrt{n}}\right]+g_{s} n \cos ^{2} \theta_{d}+g_{n} n .
\end{aligned}
$$

As shown in Table I, at $g_{s}=-g_{n} / 2$,

$$
\theta_{d}(x)=2 \arctan e^{x / \ell},
$$

where $\ell=\hbar / \sqrt{4\left|g_{s}\right| M n_{b}}$ as introduced earlier. Away from the exactly solvable point, we assume that the expression of $\theta_{d}(x)$ in Eq. (7) remains a good approximation. In other words, $\mathbf{F} / n(x)$ is assumed to take the same form as at the exactly solvable point. The reason for this will become clear later.

Let us examine the asymptotic form of Eq. (6b) far away from the core $x=0$. Assuming that $g(x) \equiv\left[n(x)-n_{b}\right] /\left(4 n_{b}\right)$ decays slower than $\left(\partial_{x} \theta_{d}\right)^{2} \sim e^{-2 x / \ell}$ for large $x \gg \ell$ [there is no solution for $g(x)$ decaying faster than $e^{-2 x / \ell}$, in the large $x$ limit, the dominant part of Eq. (6b) reads

$$
\left(g_{n}+g_{s}\right) g(x)+\ell^{2} g_{s} g^{\prime \prime}(x)=0,
$$

having a solution $g(x \gg \ell) \sim e^{-x / \ell_{d}}$, where $\ell_{d}=\lambda \ell$ is the effective density length scale. Combining the asymptotic behavior of $n(x)$ at large $x$ and $n(x)$ being an even function, it is natural to propose the ansatz (5a). The coefficients $a_{1}$ and $b_{1}$ are introduced to adjust the core structure and are determined by requiring that $n(x)$ satisfies Eq. (6) to leading order as $x \rightarrow 0$. The working assumption to obtain Eq. (5a) is $\ell_{d}>\ell(\lambda>1 / 2)$, implying $g_{n}+5 g_{s}<0$, which sets the parameter range for the solution in Eq. (5a) to be applicable. This regime includes the exactly solvable point, $g_{s}=-g_{n} / 2$ with $\lambda=1$, where $\ell_{d}=\ell$, and a single length scale describes the spin and density character of the MDW [here (5a) reduces to Eq. (4)]. In this strong spin-interaction regime (5a), the density variation near the core is important. The excitation breaks down at $g_{s}+g_{n}=0$, which is the parameter boundary of the ferromagnetic phase $[19,20]$.

In the opposite limit, where $\left|g_{s} / g_{n}\right| \ll 1$, the quantum pressure term $\sim \partial_{x}^{2} \sqrt{n} / \sqrt{n}$ becomes less important and can be 
neglected. Hence, Eq. (6b) becomes an algebraic equation of $n(x)$ with solution given Eq. (5b). The parameters $b_{1}$ and $g_{1}$ are introduced to solve Eqs. (6) near the core $x=0$ to leading order. The crossover to the weak spin-interaction regime $(5 \mathrm{~b})$ occurs at $g_{n}+5 g_{s}=0$ where $\lambda=1 / 2$, given by matching the density widths $\ell / 2$ and $\ell_{d}$. For comparison, we calculate numerical MDW results using a gradient flow method $[43,44]$. The analytical and numerical results in Figs. 1(b) and 1(c) show excellent agreement.

Let us now provide a self-consistent reasoning to explain why $\theta_{d}(x)$ in Eq. (7) serves a good approximation in the whole parameter range. First of all, it captures the main feature of the domain wall in the strongly interacting regime where the exact solution Eq. (7) is found. On the other hand, in the weak-interaction limit $\left(\left|g_{s} / g_{n}\right| \ll 1\right)$, the density $n$ can be approximated as a constant $\left(n \simeq n_{b}\right)$ and the energy density becomes

$$
\mathcal{H}=-\frac{\hbar^{2} n}{8 M}\left|\nabla \theta_{d}\right|^{2}+\frac{g_{n}}{2} n_{b}-\frac{1}{2} g_{s} n_{b}^{2} \cos ^{2} \theta_{d} .
$$

A local minimum of the energy density, determined by $\delta \mathcal{H} / \delta \theta_{d}=0$, leads to the elliptic sine-Gordon equation $\hbar^{2} /(2 M) \nabla^{2}\left(2 \theta_{d}\right)+2 n_{b} g_{s} \sin \left(2 \theta_{d}\right)=0$, having the solution $\theta_{d}=2 \arctan e^{x / \ell}$.

Since the magnetization vanishes at the core, there is no spin current across the MDW. However, the nematic tensor current is nontrivial [45]. The component number currents vary for different degenerate states. For example, with reference to the states in Table I: The component currents are zero for the vector soliton, while for SGS there are internal currents near the core that behave analogously to Josephson currents [46].

\section{FINITE MAGNETIC FIELDS}

A magnetic field along the $z$ axis breaks the $\mathrm{SO}(3)$ symmetry and the degeneracy of states presented in Table I is lifted. For $q>0$, the ground state magnetization prefers to be transverse, realizing an easy-plane ferromagnetic phase that possesses a remnant $\mathrm{SO}(2)$ symmetry $[19,20]$. Here, the SGS and the binary density domain wall are no longer stationary solutions. The type-I vector soliton is energetically favored, and exists, with some modifications, in the whole easy-plane phase $\left(0<q<2\left|g_{s}\right| n_{b}\right)$. At $g_{s}=-g_{n} / 2$, the exact solution is

$$
\begin{aligned}
\mathbf{F} & =n_{b} \sqrt{1-\tilde{q}^{2}} \tanh \left(\frac{x}{2 \ell_{q}}\right) \hat{\mathbf{e}}_{\rho}, \\
n(x) & =n_{b}\left[1-\frac{1-\tilde{q}}{2} \operatorname{sech}^{2}\left(\frac{x}{2 \ell_{q}}\right)\right],
\end{aligned}
$$

where $\tilde{q}=-q / 2 g_{s} n_{b}, \ell_{q}=\ell / \sqrt{1-\tilde{q}}$, and $\hat{\mathbf{e}}_{\rho}$ is a unit vector in the $x y$ plane. The corresponding wave function reads $\psi_{ \pm 1}=\sqrt{n_{b}(1-\tilde{q}) / 4} \tanh \left(x / 2 \ell_{d}\right)$, and $\psi_{0}=\sqrt{n_{b}(1+\tilde{q}) / 2}$. An example of a $q \neq 0$ result $\left(\hat{\mathbf{e}}_{\rho}=\hat{\mathbf{x}}\right)$ is shown in Fig. 1 .

\section{STANDING WAVES}

A conspicuous feature of the 2D dynamics of the MDW is that it is stable against transverse deformations, strikingly different from other domain walls/solitons [5-7,9-15], which decay unavoidably via snake instability. We consider easy-

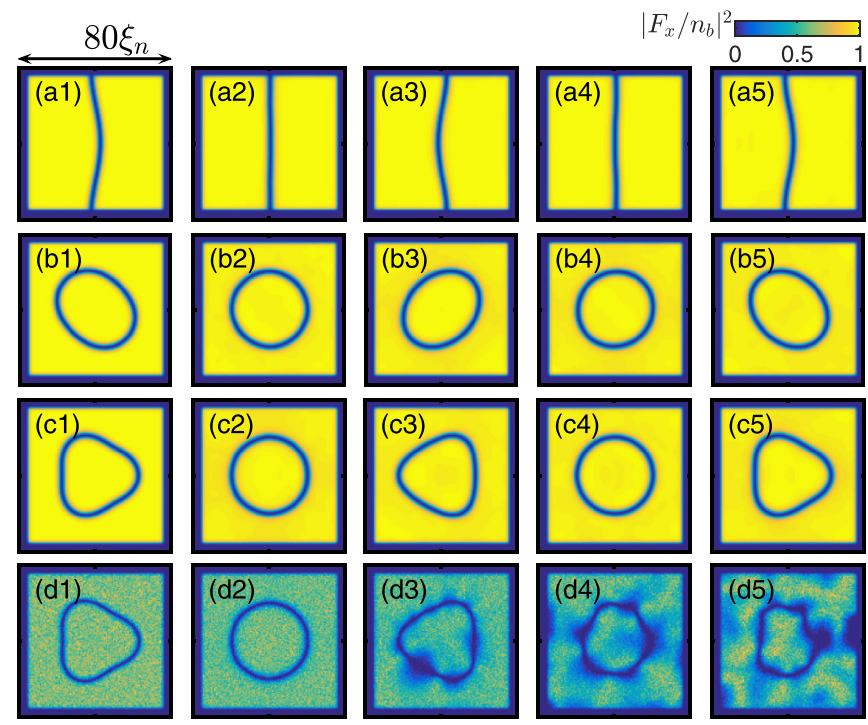

FIG. 2. One period of evolution for standing wave deformations of a MDW confined by hard-wall potentials in a square domain $x, y \in[-L, L]$ with $L=40 \xi_{n}$. The equilibrium configurations are shown in the second and fourth columns. (a1)-(a5) A standing wave on an open MDW with free end-points attached on the hard-wall boundaries; the initial configuration (MDW core location) is $x=$ $A \cos (\pi y / L), y \in[-L, L]$ with $L=40 \xi_{n}$ and $A=2.5 \xi_{n}$. (b1)-(b5), (c1)-(c5) Standing waves on a closed MDW. The initial configurations are determined by $R_{0}-\sqrt{x^{2}+y^{2}}+A \sin [s \arctan (y / x)]=0$ where $R_{0}=L / 2, s=2$ (dipole mode) for (b1) and $s=3$ (triple mode) for (c1). Oscillation periods $T$ are: (a) $T \simeq 620 t_{0}$; (b) $T \simeq$ $620 t_{0}$; (c) $T \simeq 300 t_{0}$, where $t_{0}=\hbar / g_{n} n_{b}$ and $g_{s} / g_{n}=-0.1$. (d1)(d5) Results with white noise added to the initial condition of (c1), causing a $\sim 1 \%$ increase in particle number. Such stability also stands for the open domain walls. Note that for (a), (b), and (c), $\left|F_{x}\right|=|\mathbf{F}|$.

plane domains with $\mathbf{F}$ along the $x$ axis and two fundamental static MDW geometries in the $x-y$ plane for $q=0$ : closed circle and open straight line with endpoints attached on the boundaries [47] (see Fig. 2). We excite standing waves on these static MDWs by deforming them transversely. The subsequent time evolution shown in Figs. 2(a)-2(c) is periodic and resembles harmonic modes vibrations [48]. Our simulations [49] also show that the standing waves persist without decay, combined with internal spin-exchange dynamics between components of the wave function (see Fig. 6 in Appendix B). During the time evolution, $F_{y}$ and $F_{z}$ remain zero, the magnetization conservation manifests itself as a geometrical constraint of the domain-wall motion: The area enclosed by the domain wall remains unchanged. There is no spin current crossing the MDW. The enclosed regions form magnetic bubbles, inside which the magnetization $F_{x}$ has the opposite orientation from the outer one and such feature remains in the presence of noise (see Figs. 2 and 3). Consequently, propagating open MDWs and expanding/shrinking closed MDWs are prohibited, becoming possible when applying magnetic fields along the $z$ axis.

\section{DYNAMICAL INSTABILITY}

Here, we systematically study the stability of the MDW by means of Bogoliubov-de Gennes equations (BdGs). Let 


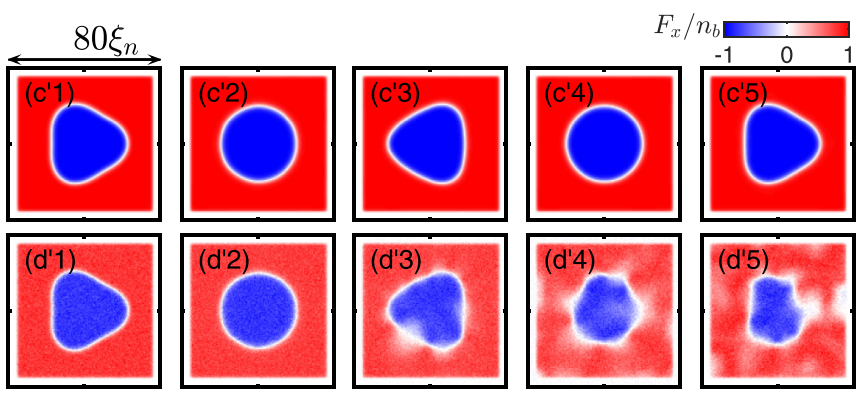

FIG. 3. The transverse magnetization $F_{x}$ during the time evolution. $\left(\mathrm{c}^{\prime} 1\right)-\left(\mathrm{c}^{\prime} 5\right)$ and $\left(\mathrm{d}^{\prime} 1\right)-\left(\mathrm{d}^{\prime} 5\right)$ correspond to Fig. 2(c1)-(c5) and (d1)-(d5), respectively.

us consider a straight infinitely long MDW along the $y$ axis located in the middle of a slab of width $L_{s} \gg \ell$. Denoting the stationary MDW as $\psi_{s}$, we consider a perturbation $\delta \psi=$ $u(\mathbf{r}) e^{-i \omega t}+v^{*}(\mathbf{r}) e^{i \omega^{*} t}$. Linearizing about $\psi=\psi_{s}+\delta \psi$ in Eq. (2) yields the BdG equations

$$
\hbar \omega\left(\begin{array}{l}
u \\
v
\end{array}\right)=\left(\begin{array}{cc}
\mathcal{L}_{\mathrm{GP}}+X-\mu & \Delta \\
-\Delta^{*} & -\left(\mathcal{L}_{\mathrm{GP}}+X-\mu\right)^{*}
\end{array}\right)\left(\begin{array}{l}
u \\
v
\end{array}\right),
$$

where the stationary wave function satisfies $\mathcal{L}_{\mathrm{GP}} \psi_{s}=$ $\mu \psi_{s}, \quad X=g_{s} \sum_{v} S_{v} \psi_{s} \psi_{s}^{\dagger} S_{v}+g_{n} \psi_{s} \psi_{s}^{\dagger}, \quad \Delta=g_{n} \psi_{s} \psi_{s}^{T}+$ $g_{s} \sum_{v}\left(S_{v} \psi_{s}\right)\left(S_{v} \psi_{s}\right)^{T}$ and $\mu=\left(g_{n}+g_{s}\right) n_{b}+q / 2$. The translational symmetry along $y$ allows us to parametrize the perturbations with the wave vector $k_{y}$ as $u(\mathbf{r})=u(x) e^{i k_{y} y}$ and $v(\mathbf{r})=v(x) e^{i k_{y} y}$. We numerically solve Eq. (12) with Neumann boundary conditions at $x$-axis boundaries [51], and find two modes with an imaginary energy [Fig. 4(a)], marking a dynamical instability in the system (a mode that grows exponentially with time).

Different from the snake instability [6], the imaginary part of the excitation energy $\operatorname{Im}(\omega)$ does not vanish as $k_{y} \rightarrow 0$, but instead approaches a finite value [Fig. 4(a)], implying that this instability also exists in 1D. $F_{x}$ is unchanged as the unstable mode grows, however it causes the unmagnetized core of the MDW to develop a $F_{y}$-texture of wavelength $\pi / k_{y}$. This corresponds to the formation of a chain of "magnetic vortex" cores [52] at the nodes of this texture [Fig. 4(b)].

The $k_{y}$ range of unstable modes and the magnitude of the imaginary energy is largest at intermediate values of $g_{s} / g_{n}$, and increases with increasing $q$ [see inset to Fig. 4(a)]. Based on the magnetic texture created by the unstable mode, we refer to it as spin-twist instability. In dynamics, the growth of this instability leads to spin waves of $F_{y}$ and $F_{z}$ while the topological structure of the MDW in $F_{x}$ remains unchanged, consistent with the noisy dynamics observed in Fig. 2(d). Note that this characteristic feature does not rely on the conservation law of magnetization, and holds in the presence of magnetic fields $(q>0)$. The growth of $F_{y}$ and $F_{z}$ could be stabilizing the domain wall by absorbing the perturbations, in analogy to a buffering effect.

\section{CONCLUSIONS AND OUTLOOK}

We found a novel magnetic domain wall in a quasi-2D ferromagnetic spin-1 BEC that is stable against the snake
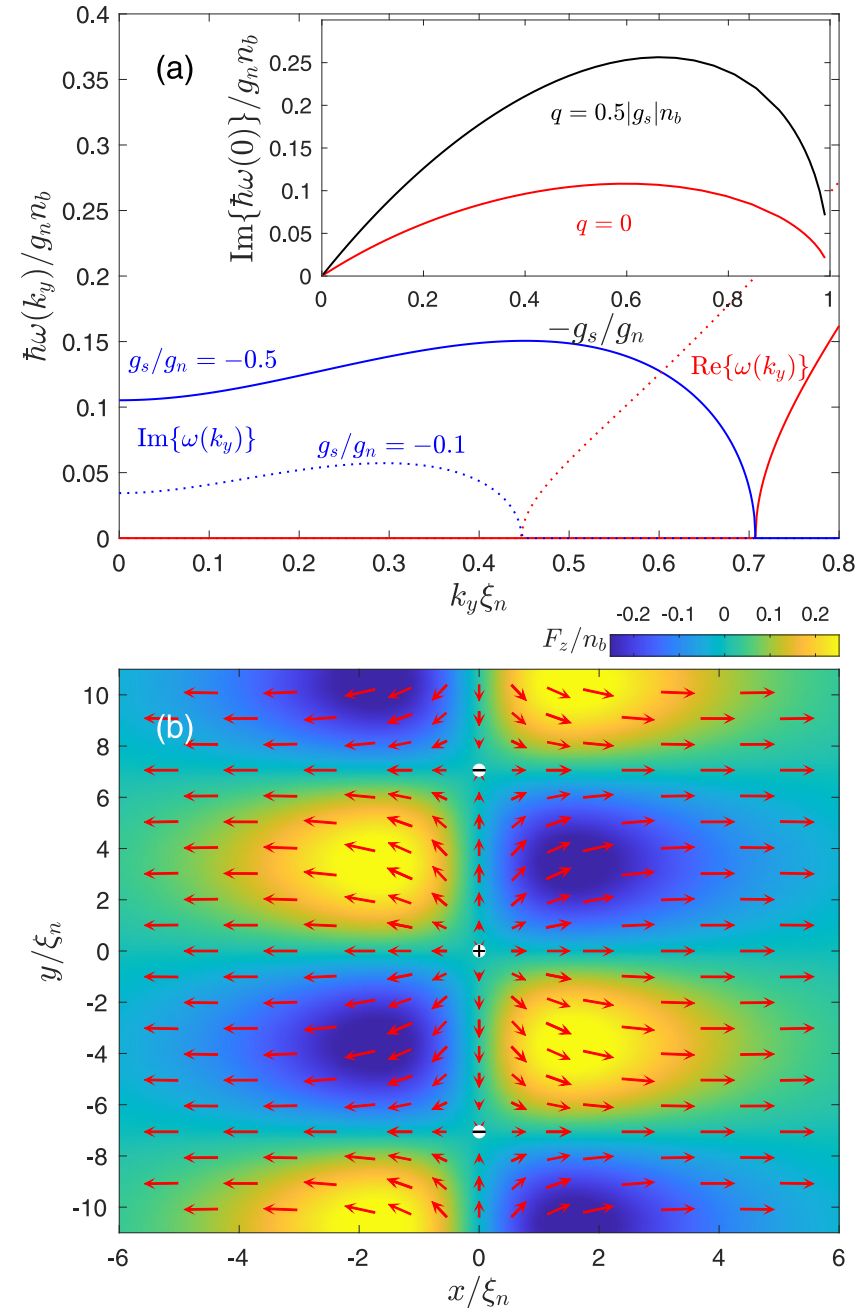

FIG. 4. Unstable spin-twist modes. (a) Spectrum of the unstable modes for $q=0$ and two values of $g_{s} / g_{n}$. For $g_{s} / g_{n}=-1 / 2$, the bifurcation point $(\omega=0)$ occurs exactly at $k_{y} \xi_{n}=1 / \sqrt{2}$. Inset shows the magnitude of the long wavelength instability as $g_{s} / g_{n}$ varies. (b) The spin-texture created by the unstable mode [50] at $k_{y} \xi_{n} \simeq 0.445$ where the maximum imaginary frequency is reached for $q=0$ and $g_{s} / g_{n}=-1 / 2$. White circles with + and - indicating positive and negative circulation spin-vortices, respectively. The red arrows and the background color represent transverse magnetization field $\left(F_{x}, F_{y}\right)$ and longitudinal magnetization $F_{z}$, respectively.

instability and white noise. Along with the exact solutions, an accurate analytic solution applicable to the whole ferromagnetic phase has also been developed. Through the underlying symmetries of the spin-1 system, we have shown that various distinct nonlinear structures such as the sine-Gordon soliton, vector solitons and an immiscible binary density domain wall occurring in unrelated binary systems are unified into the magnetic domain wall. Our findings open a possibility to study rich 2D dynamics of domain-wall solitons, could be important for determining the universality class of the ferromagnetic phase transition of 2D spin-1 BECs at finite temperature [53,54], coarsening dynamics involving both spin order and superfluid order $[29,55]$ and dynamics of stretched polar-core vortices $[56,57]$. 
It will be feasible to observe magnetic domain walls in current experiments with ferromagnetic spinor BECs. The necessary techniques for manipulating the spin degrees of freedom of a spin-1 BEC [14], and for nondestructively measuring its spin dynamics [22] have already been demonstrated. Coupled with a planar or flat-bottom optical trap (e.g., $[58,59])$ opens the possibility for investigating of domain-wall dynamics. Most work with ferromagnetic spin-1 BECs to date has been conducted with ${ }^{87} \mathrm{Rb}$, which has $-g_{s} / g_{n} \sim 10^{-2}$ and is in the weakly spin-interacting regime. However, recently a ${ }^{7} \mathrm{Li}$ spin-1 BEC has been prepared with $-g_{s} / g_{n} \sim 0.5$ [60], thus in the strong spin-interacting regime close to the exactly solvable point.

\section{ACKNOWLEDGMENTS}

We thanks M. Antonio, T. Luke, L. A. Williamson, Russell Bisset, and Danny Baillie for useful discussions. X.Y. acknowledges the support from NSAF through Grant No. U1930403. P.B.B. acknowledges support from the Marsden Fund of the Royal Society of New Zealand.

\section{APPENDIX A: SPIN-1 GROSS-PITAEVSKII EQUATIONS IN HYDRODYNAMICAL VARIABLES}

In terms of the hydrodynamical variables $\left\{n, n_{d}, \theta_{d}, \theta_{s}\right\}$, the stationary GPE for $q=0$ becomes

$$
\begin{aligned}
0= & -\frac{\hbar^{2}}{2 M} \nabla \cdot\left(n \nabla \theta_{s}+n_{d} \nabla \theta_{d}\right), \\
0= & \frac{\hbar^{2}}{2 M} \nabla \cdot\left(n \nabla \theta_{d}+n_{d} \nabla \theta_{s}\right)+g_{s}\left(n^{2}-n_{d}^{2}\right) \sin \left(2 \theta_{d}\right),(\mathrm{A} 1 \mathrm{~b}) \\
0= & -\frac{\hbar^{2}}{2 M}\left[\frac{1}{\sqrt{n+n_{d}}} \nabla^{2} \sqrt{n+n_{d}}+\frac{1}{\sqrt{n-n_{d}}} \nabla^{2} \sqrt{n-n_{d}}\right. \\
& \left.-\frac{1}{2}\left(\left|\nabla \theta_{s}\right|^{2}+\left|\nabla \theta_{d}\right|^{2}\right)\right]+g_{s} n \cos \left(2 \theta_{d}\right) \\
& +\left(g_{s}+2 g_{n}\right) n-2 \mu, \\
0= & \frac{\hbar^{2}}{2 M}\left[\frac{1}{\sqrt{n+n_{d}}} \nabla^{2} \sqrt{n+n_{d}}-\frac{1}{\sqrt{n-n_{d}}} \nabla^{2} \sqrt{n-n_{d}}\right. \\
& -\nabla \alpha \cdot \nabla \beta]+g_{s} n_{d} \cos (2 \beta)+g_{s} n_{d},
\end{aligned}
$$

where $n_{d}=2 n_{ \pm 1}-n_{0}, \theta_{s}=\theta_{ \pm 1}+\theta_{0}, \theta_{d}=\theta_{ \pm 1}-\theta_{0}, n=$ $n_{+}+n_{-}+n_{0}, \mu=\left(g_{n}+g_{s}\right) n_{b}$ is the chemical potential and $n_{b}$ is the ground state total number density. Here we assume that $\theta_{+1}=\theta_{-1}$ and $n_{+1}=n_{-1}$.

\section{APPENDIX B: REAL-TIME EVOLUTION}

Here we present further evidence of the stability. Figure 3 shows that the topological nature of the MDW, i.e., the $\pi$ phase jump across the core, is well preserved during the domain-wall motion. This can be also seen in Fig. 5(a), which shows the profile of the transverse magnetization $F_{x}(x, y=0)$ at different times.
In our simulations, the box potential takes the following form:

$$
\begin{aligned}
V(x, y)= & V_{0}\left\{2-\tanh \left[\left(\frac{L}{c}-|x|\right) b\right]\right. \\
& \left.-\tanh \left[\left(\frac{L}{c}-|y|\right) b\right]\right\},
\end{aligned}
$$

where $x, y \in[-L, L], L$ is the box size, $V_{0} \gg \mu$ is the hight of the potential barrier, $b \sim \xi_{n}^{-1}$ is the width and $c$ should be chosen slightly greater than one.

The standing wave excitation on the MDW can last a long time without decay. In order to quantify this property, we
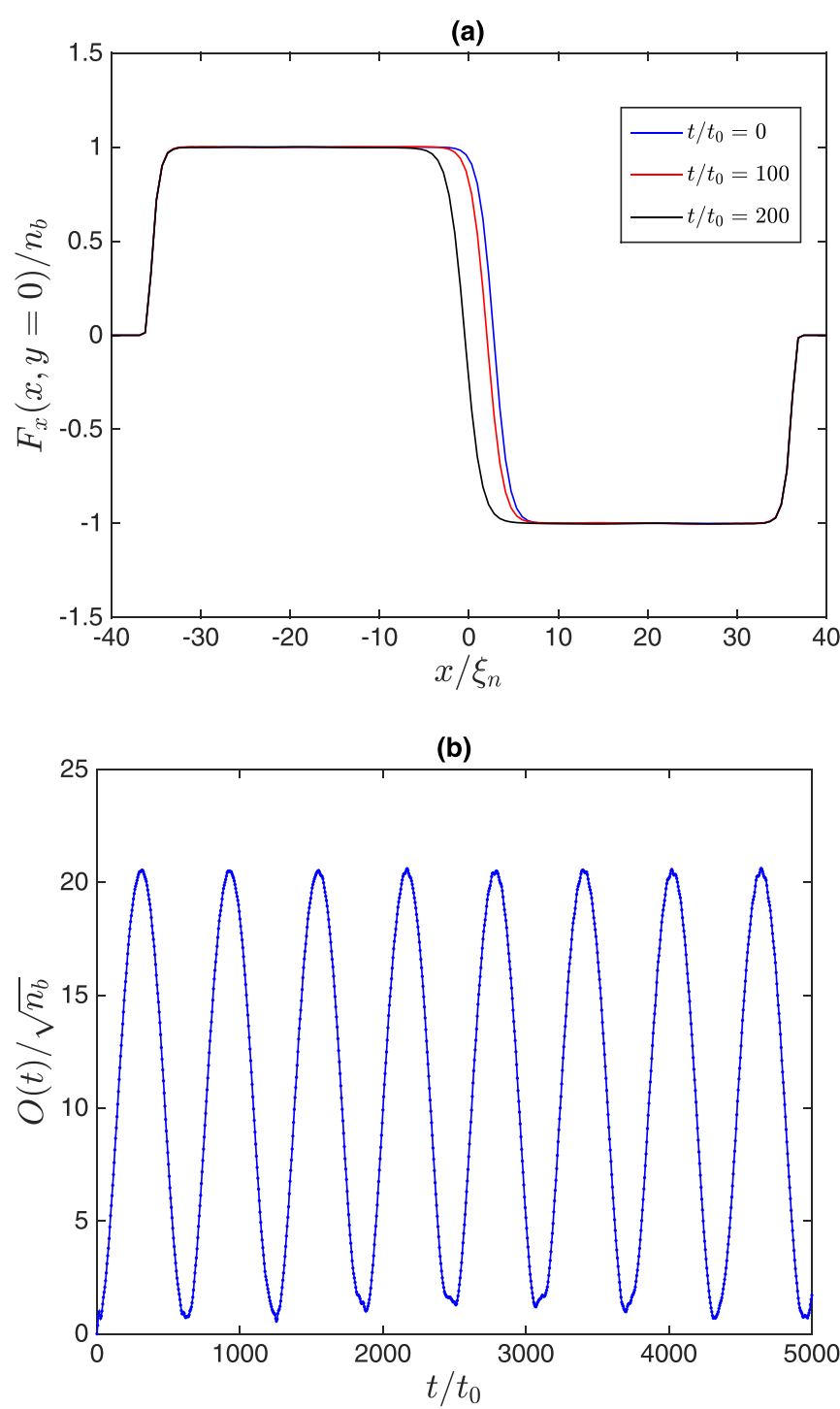

FIG. 5. (a) Shows the spin-density cross section $F_{x}(x, y=0)$ of the open MDW shown in Fig. 2(a) at different times. The solitonlike profile of the magnetization is preserved during the motion. (b) Shows the periodic behavior of the overlap function for the open domain-wall configuration [Fig. 2(a)], demonstrating that the standing wave on the MDW persists without decay over long time periods. 


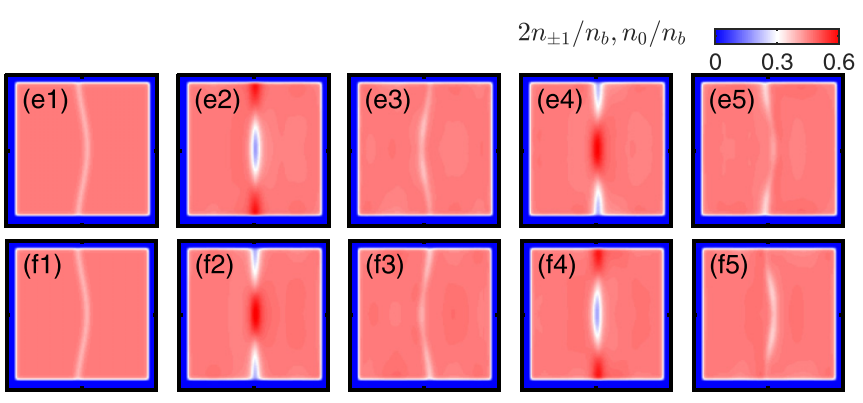

FIG. 6. Spin-exchange dynamics during the MDW oscillation shown in Fig. 2(a1)-(a5). (e1)-(e5), (f1)-f(5) show dynamics of component densities $2 n_{ \pm 1}$ and $n_{0}$, respectively. introduce an overlap function

$$
O(t) \equiv\left(\int d^{2} \mathbf{r}\left|F_{x}(\mathbf{r}, t)-F_{x}(\mathbf{r}, 0)\right|^{2}\right)^{1 / 2}
$$

that measures the overlap of transverse magnetization profile at time $t$ with its initial profile. Figure 5(b) shows the periodic behavior of $O(t)$ for the open domain wall [Fig. 2(a)], revealing that the standing wave on the MDW persists without decay.

It is worthwhile to mention that along with the domain-wall oscillation, components of the wave function exhibit spinexchange dynamics [Fig. 6].
[1] Y. M. Bunkov and H. Godfrin, Topological Defects and the Non-equilibrium Dynamics of Symmetry Breaking Phase Transitions (Springer Science \& Business Media, Dordrecht, 2012), Vol. 549.

[2] N. Manton and P. Sutcliffe, Topological Solitons (Cambridge University Press, Cambridge, 2004).

[3] M. Eto, Y. Hirono, M. Nitta, and S. Yasui, Vortices and other topological solitons in dense quark matter, Prog. Theor. Exp. Phys. 2014, 012D01 (2014).

[4] A. Vilenkin and E. P. S. Shellard, Cosmic Strings and Other Topological Defects (Cambridge University Press, Cambridge, 2000).

[5] E. A. Kuznetsov and S. K. Turitsyn, Instability and collapse of solitons in media with a defocusing nonlinearity, Zh. Eksp. Teor. Fiz. 94, 129 (1988).

[6] A. E. Muryshev, H. B. van Linden van den Heuvell, and G. V. Shlyapnikov, Stability of standing matter waves in a trap, Phys. Rev. A 60, R2665 (1999).

[7] B. P. Anderson, P. C. Haljan, C. A. Regal, D. L. Feder, L. A. Collins, C. W. Clark, and E. A. Cornell, Watching Dark Solitons Decay into Vortex Rings in a Bose-Einstein Condensate, Phys. Rev. Lett. 86, 2926 (2001).

[8] G. Huang, V. A. Makarov, and M. G. Velarde, Two-dimensional solitons in Bose-Einstein condensates with a disk-shaped trap, Phys. Rev. A 67, 023604 (2003).

[9] D. T. Son and M. A. Stephanov, Domain walls of relative phase in two-component Bose-Einstein condensates, Phys. Rev. A 65, 063621 (2002).

[10] K. Ihara and K. Kasamatsu, Transverse instability and disintegration of a domain wall of a relative phase in coherently coupled two-component Bose-Einstein condensates, Phys. Rev. A 100, 013630 (2019).

[11] A. Gallemí, L. P. Pitaevskii, S. Stringari, and A. Recati, Decay of the relative phase domain wall into confined vortex pairs: The case of a coherently coupled bosonic mixture, Phys. Rev. A 100, 023607 (2019).

[12] C. Qu, L. P. Pitaevskii, and S. Stringari, Magnetic Solitons in a Binary Bose-Einstein Condensate, Phys. Rev. Lett. 116, 160402 (2016).

[13] A. Farolfi, D. Trypogeorgos, C. Mordini, G. Lamporesi, and G. Ferrari, Observation of Magnetic Solitons in Two-Component Bose-Einstein Condensates, Phys. Rev. Lett. 125, 030401 (2020).
[14] X. Chai, D. Lao, K. Fujimoto, R. Hamazaki, M. Ueda, and C. Raman, Magnetic Solitons in a Spin-1 Bose-Einstein Condensate, Phys. Rev. Lett. 125, 030402 (2020).

[15] S. Kang, S. W. Seo, H. Takeuchi, and Y. Shin, Observation of Wall-Vortex Composite Defects in a Spinor Bose-Einstein Condensate, Phys. Rev. Lett. 122, 095301 (2019).

[16] T.-L. Ho, Spinor Bose Condensates in Optical Traps, Phys. Rev. Lett. 81, 742 (1998).

[17] T. Ohmi and K. Machida, Bose-Einstein condensation with internal degrees of freedom in alkali atom gases, J. Phys. Soc. Jpn. 67, 1822 (1998).

[18] L. E. Sadler, J. M. Higbie, S. R. Leslie, M. Vengalattore, and D. M. Stamper-Kurn, Spontaneous symmetry breaking in a quenched ferromagnetic spinor Bose-Einstein condensate, Nature 443, 312 (2006).

[19] D. M. Stamper-Kurn and M. Ueda, Spinor Bose gases: Symmetries, magnetism, and quantum dynamics, Rev. Mod. Phys. 85, 1191 (2013).

[20] Y. Kawaguchi and M. Ueda, Spinor Bose-Einstein condensates, Phys. Rep. 520, 253 (2012)

[21] W. Zhang, D. L. Zhou, M.-S. Chang, M. S. Chapman, and L. You, Dynamical Instability and Domain Formation in a Spin-1 Bose-Einstein Condensate, Phys. Rev. Lett. 95, 180403 (2005).

[22] J. M. Higbie, L. E. Sadler, S. Inouye, A. P. Chikkatur, S. R. Leslie, K. L. Moore, V. Savalli, and D. M. Stamper-Kurn, Direct Nondestructive Imaging of Magnetization in a Spin-1 BoseEinstein Gas, Phys. Rev. Lett. 95, 050401 (2005).

[23] H. Saito and M. Ueda, Spontaneous magnetization and structure formation in a spin-1 ferromagnetic Bose-Einstein condensate, Phys. Rev. A 72, 023610 (2005).

[24] H. Saito, Y. Kawaguchi, and M. Ueda, Topological defect formation in a quenched ferromagnetic Bose-Einstein condensates, Phys. Rev. A 75, 013621 (2007).

[25] W. Zhang, Ö. E. Müstecaplıoğlu, and L. You, Solitons in a trapped spin-1 atomic condensate, Phys. Rev. A 75, 043601 (2007).

[26] M. Vengalattore, S. R. Leslie, J. Guzman, and D. M. StamperKurn, Spontaneously Modulated Spin Textures in a Dipolar Spinor Bose-Einstein Condensate, Phys. Rev. Lett. 100, 170403 (2008).

[27] Y. Kawaguchi, H. Saito, K. Kudo, and M. Ueda, Spontaneous magnetic ordering in a ferromagnetic spinor dipolar BoseEinstein condensate, Phys. Rev. A 82, 043627 (2010). 
[28] L. A. Williamson and P. B. Blakie, Universal Coarsening Dynamics of a Quenched Ferromagnetic Spin-1 Condensate, Phys. Rev. Lett. 116, 025301 (2016).

[29] M. Prüfer, P. Kunkel, H. Strobel, S. Lannig, D. Linnemann, C.-M. Schmied, J. Berges, T. Gasenzer, and M. K. Oberthaler, Observation of universal dynamics in a spinor Bose gas far from equilibrium, Nature 563, 217 (2018).

[30] H. E. Nistazakis, D. J. Frantzeskakis, P. G. Kevrekidis, B. A. Malomed, and R. Carretero-González, Bright-dark soliton complexes in spinor Bose-Einstein condensates, Phys. Rev. A 77, 033612 (2008).

[31] Th. Busch and J. R. Anglin, Dark-Bright Solitons in Inhomogeneous Bose-Einstein Condensates, Phys. Rev. Lett. 87, 010401 (2001).

[32] T. M. Bersano, V. Gokhroo, M. A. Khamehchi, J. D’Ambroise, D. J. Frantzeskakis, P. Engels, and P. G. Kevrekidis, ThreeComponent Soliton States in Spinor $f=1$ Bose-Einstein Condensates, Phys. Rev. Lett. 120, 063202 (2018).

[33] S. V. Manakov, On the theory of two-dimensional stationary self-focusing of electromagnetic waves, Sov. Phys. JETP 38, 248 (1974).

[34] The size of the system in $z$ direction is much smaller than the spin healing length $\xi_{s}=\hbar / \sqrt{2\left|g_{s}\right| M n_{b}}$.

[35] Generators of the rotational group $\mathrm{SO}(3)$ :

$$
\begin{aligned}
S_{x} & =\frac{1}{\sqrt{2}}\left(\begin{array}{ccc}
0 & 1 & 0 \\
1 & 0 & 1 \\
0 & 1 & 0
\end{array}\right), \quad S_{y}=\frac{i}{\sqrt{2}}\left(\begin{array}{ccc}
0 & -1 & 0 \\
1 & 0 & -1 \\
0 & 1 & 0
\end{array}\right), \\
S_{z} & =\left(\begin{array}{ccc}
1 & 0 & 0 \\
0 & 0 & 0 \\
0 & 0 & -1
\end{array}\right)
\end{aligned}
$$

[36] $F_{x}=\left(\psi_{0}^{*} \psi_{-1}+\psi_{0} \psi_{+1}^{*}+\right.$ H.c $) / \sqrt{2} ; \quad F_{y}=\left[\psi_{0}^{*}\left(\psi_{-1}-\psi_{+1}\right)-\right.$ H.c] $/ \sqrt{2} \mathrm{i} ; F_{z}=n_{+1}-n_{-1}$.

[37] In terms of scattering length, the exactly solvable point corresponds to $4 a_{2}=a_{0}$, where $a_{F}$ is the s-wave scattering length in the total spin $F$ channel. Here we used the following relation $g_{n}=4 \pi \hbar^{2}\left(a_{0}+2 a_{2}\right) / 3 M$ and $g_{s}=4 \pi \hbar^{2}\left(a_{2}-a_{0}\right) / 3 M$ [16].

[38] In BEC experiments, the drawback of introducing Rabicoupling (magnetic noise) sometimes can be problematic [61,62].

[39] P. Ao and S. T. Chui, Binary Bose-Einstein condensate mixtures in weakly and strongly segregated phases, Phys. Rev. A 58, 4836 (1998)

[40] B. A. Malomed, A. A. Nepomnyashchy, and M. I. Tribelsky, Domain boundaries in convection patterns, Phys. Rev. A 42, 7244 (1990).

[41] Only in the Manakov limit, the two-component BEC possesses SU(2) symmetry.

[42] X. Chai, Di Lao, K. Fujimoto, and C. Raman, Magnetic soliton: From two to three components with $\mathrm{SO}(3)$ symmetry, Phys. Rev. Research 3, L012003 (2021).

[43] F. Y. Lim and W. Bao, Numerical methods for computing the ground state of spin-1 Bose-Einstein condensates in a uniform magnetic field, Phys. Rev. E 78, 066704 (2008).
[44] W. Bao and F. Y. Lim, Computing ground states of spin-1 BoseEinstein condensates by the normalized gradient flow, SIAM J. Sci. Comput. 30, 1925 (2008).

[45] The details will be discussed elsewhere.

[46] A. Barone and G. Paterno, Physics and Applications of the Josephson Effect (Wiley, New York, 1982).

[47] Configurations with free ends are unstable.

[48] For large deformations of the magnetic domain wall from its equilibrium position the motion is periodic but is not harmonic.

[49] L. M. Symes, R. I. McLachlan, and P. B. Blakie, Efficient and accurate methods for solving the time-dependent spin-1 GrossPitaevskii equation, Phys. Rev. E 93, 053309 (2016).

[50] The BdG equation is $G(u, v)^{T}=E(u, v)^{T}$, where $G$ is the BdG operator. The symmetry $\sigma_{x} G \sigma_{x}=-G^{*}$, with $\sigma_{x}$ the $x$ Pauli matrix, ensures that $\left(v^{*}, u^{*}\right)^{T}$ is an eigenstate with eigenvalue $-E^{*}$. For the unstable modes, $\operatorname{Re}(E)=0$, hence $-E^{*}=E$. Then any linear combination of $(u, v)^{T}$ and $\left(v^{*}, u^{*}\right)^{T}$ is an eigenstate of $G$. Here the unstable modes are chosen to be real, in which case the two unstable modes are identical.

[51] The unstable modes must be localized in space and are insensitive to boundary conditions as long as $L_{s} \gg \ell$.

[52] In $2 \mathrm{D}$ vector fields with three components, winding number is not well defined and hence the terminology vortex is quoted.

[53] A. J. A. James and A. Lamacraft, Phase Diagram of TwoDimensional Polar Condensates in a Magnetic Field, Phys. Rev. Lett. 106, 140402 (2011).

[54] M. Kobayashi, Berezinskii-Kosterlitz-Thouless transition of spin-1 spinor Bose gases in the presence of the quadratic Zeeman effect, J. Phys. Soc. Jpn. 88, 094001 (2019).

[55] A. Bourges and P. B. Blakie, Different growth rates for spin and superfluid order in a quenched spinor condensate, Phys. Rev. A 95, 023616 (2017).

[56] L. A. Williamson and P. B. Blakie, Dynamics of polar-core spin vortices in a ferromagnetic spin-1 Bose-Einstein condensate, Phys. Rev. A 94, 063615 (2016).

[57] A. M. Turner, Mass of a Spin Vortex in a Bose-Einstein Condensate, Phys. Rev. Lett. 103, 080603 (2009).

[58] L. Chomaz, L. Corman, T. Bienaimé, R. Desbuquois, C. Weitenberg, S. Nascimbène, J. Beugnon, and J. Dalibard, Emergence of coherence via transverse condensation in a uniform quasi-two-dimensional Bose gas, Nat. Commun. 6, 6162 (2015).

[59] G. Gauthier, I. Lenton, N. McKay Parry, M. Baker, M. J. Davis, H. Rubinsztein-Dunlop, and T. W. Neely, Direct imaging of a digital-micromirror device for configurable microscopic optical potentials, Optica 3, 1136 (2016)

[60] S. Huh, K. Kim, K. Kwon, and J.-Y. Choi, Observation of a strongly ferromagnetic spinor Bose-Einstein condensate, Phys. Rev. Research 2, 033471 (2020).

[61] D. Trypogeorgos, A. Valdés-Curiel, N. Lundblad, and I. B. Spielman, Synthetic clock transitions via continuous dynamical decoupling, Phys. Rev. A 97, 013407 (2018).

[62] A. Farolfi, D. Trypogeorgos, G. Colzi, E. Fava, G. Lamporesi, and G. Ferrari, Design and characterization of a compact magnetic shield for ultracold atomic gas experiments, Rev. Sci. Instrum. 90, 115114 (2019). 